Bull. Korean Math. Soc. 52 (2015), No. 2, pp. 395-408

http://dx.doi.org/10.4134/BKMS.2015.52.2.395

\title{
THE DISTINGUISHING NUMBERS \\ OF MERGED JOHNSON GRAPHS
}

\author{
Dongseok Kim, Young Soo Kwon, And Jaeun Lee
}

\begin{abstract}
In present article, we determine the distinguishing number of the merged Johnson graphs which are generalization of both the Kneser graphs and the Johnson graphs.
\end{abstract}

\section{Introduction}

The distinguishing number of a graph $G$ is the minimum number of colors for which there exists an assignment of colors to the vertices of $G$ such that the identity is the only color-preserving automorphism of $G$. Generally, for a permutation group $\Gamma$ acting on $X$, the distinguishing number of $\Gamma$ is the minimum number of cells of a partition $\pi$ of $X$ satisfying that the identity is the only element of $\Gamma$ fixing each cell of $\pi$. Albertson and Collins first introduced the distinguishing number of a graph [3] and there have been many interesting results on the distinguishing numbers of graphs and permutation groups in last few years $[1,2,3,4,5,7,8,10,11]$.

Here we consider a class of graphs based on the Johnson graphs. For positive integers $k, n$ such that $1 \leq k \leq \frac{n}{2}$, the Johnson graph $J(n, k)$ has vertices given by the $k$-subsets of $[n]=\{1,2, \ldots, n\}$ and there exists an edge between two vertices if and only if their intersection has size $k-1$. Given a nonempty subset $I \subseteq\{1,2, \ldots, k\}$, the merged Johnson graph $J(n, k)_{I}$ is the union of the distance $i$ graphs $J(n, k)_{i}$ of $J(n, k)$ for all $i \in I$, namely, two $k$-subsets are adjacent in $J(n, k)_{I}$ if and only if their intersection has $k-i$ elements for some $i \in I$. The merged Johnson graphs $J(n, k)_{I}$ include many interesting graphs such as the Johnson graph $J(n, k)=J(n, k)_{\{1\}}$ and the Kneser graph $K(n, k)=J(n, k)_{\{k\}}$.

In [2], M. O. Albertson and D. L. Boutin determined the distinguishing number of the Kneser graphs. The aim of the present article is to determine the distinguishing number of the merged Johnson graphs.

Received April 17, 2013; Revised August 23, 2013.

2010 Mathematics Subject Classification. 05C15.

Key words and phrases. distinguishing numbers, merged Johnson graphs.

This research was supported by the Yeungnam University research grants in 2012. 
The outline of this paper is as follows. In Section 2, we review some preliminaries regarding the distinguishing numbers and the merged Johnson graphs. In Section 3, we find Theorem 3.2 which addresses the distinguishing numbers of the merged Johnson graphs. We also prove lemmas which are used for a proof of the main theorem. At last, we provide a proof of Theorem 3.2 in Section 4.

\section{Preliminaries}

For a given graph $G$, a coloring $f: V(G) \rightarrow\{1,2, \ldots, r\}$ is said to be $r$-distinguishing if the identity is the only graph automorphism $\phi$ satisfying $f(\phi(v))=f(v)$ for all $v \in V(G)$. This means that the distinguishing coloring is a symmetry-breaking coloring of $G$. The distinguishing number, denoted by $\operatorname{Dist}(G)$, is the minimum $r$ that $G$ has an $r$-distinguishing coloring. One can easily see that $\operatorname{Dist}(G)=\operatorname{Dist}\left(G^{c}\right)$ where $G^{c}$ is the complement of $G$. If $G$ is an asymmetric graph, namely if the identity is the only automorphism of $G$, then $\operatorname{Dist}(G)=1$. In fact, the converse is also true.

For a graph $G$ and for a subset $S \subseteq V(G)$, a coloring $f: S \rightarrow\{1,2, \ldots, s\}$ is said to be $s$-distinguishing if for any graph automorphism $\phi$ of $G$ fixing $S$ setwisely and satisfying $f(\phi(v))=f(v)$ for all $v \in S$ fixes all elements of $S$. In this case, $\phi$ does not need to fix other vertices outside of the given set $S$. If there exists an $s$-distinguishing coloring for $S$, the set $S$ is called an $s$-distinguishable set.

For a graph $G$, a subset $S \subseteq V(G)$ is called a determining set if the identity is the only automorphism fixing every element of $S$. Note that if $S \subseteq V(G)$ is a determining set, then any subset $T \subseteq V(G)$ containing $S$ is also a determining set. The determining sets provide a useful tool for finding the distinguishing number of $G$ as stated in the following theorem.

Proposition 2.1 ([2]). For a given graph $G, G$ has an $r$-distinguishable determining set if and only if $G$ has an $(r+1)$-distinguishing coloring.

Consequently, we find the following corollary which will be used in the proof of our results.

Corollary 2.2. For a given graph $G$, if there is an asymmetric subgraph of $G$ induced by a determining set $S$, then $\operatorname{Dist}(G)=1$ or 2 .

Proof. For a graph automorphism $\phi$ of $G$ fixing $S$ set-wisely, the restriction of $\phi$ on the induced subgraph $\langle S\rangle$ is a graph automorphism of $\langle S\rangle$. Since $\langle S\rangle$ is asymmetric, the coloring $f(v)=1$ for all $v \in S$ is a 1-distinguishing. Since $S$ is a determining set, $G$ has a 2-distinguishing coloring by Proposition 2.1.

Using Proposition 2.1, M. O. Albertson and D. L. Boutin determined the distinguishing number of the Kneser graphs as follows.

Proposition $2.3([2])$. For any integers $n>k \geq 2$ with $n>2 k$, Dist $(K(n, k))$ $=2$ except $(n, k)=(5,2)$; and $\operatorname{Dist}(K(5,2))=3$. 
Note that the Kneser graph $K(5,2)$ is isomorphic to the Pertersen graph and its distinguishing number is $3 . K(n, 1)$ is isomorphic to the complete graph and so $\operatorname{Dist}(K(n, 1))=n$ for any integer $n$.

For a graph $G$, the distinguishing number $\operatorname{Dist}(G)$ is equal to the distinguishing number of $\operatorname{Aut}(G)$ which acts on the vertex set $V(G)$. Hence we have the following lemma. The proof is straightforward and we omit it.

Lemma 2.4. Let $G_{1}$ and $G_{2}$ be two graphs having the same vertex set $V$.

(1) If $\operatorname{Aut}\left(G_{1}\right)$ is a subgroup of $\operatorname{Aut}\left(G_{2}\right)$ as acting groups on $V$, then $\operatorname{Dist}\left(G_{1}\right) \leq \operatorname{Dist}\left(G_{2}\right)$.

(2) If $\operatorname{Aut}\left(G_{1}\right)=\operatorname{Aut}\left(G_{2}\right)$ as acting groups on $V$, then $\operatorname{Dist}\left(G_{1}\right)=\operatorname{Dist}\left(G_{2}\right)$.

\section{The distinguishing numbers of the merged Johnson graphs}

Let $\Omega$ be the set of all $k$-subsets of $[n]$. The action of $S_{n}$ on $[n]$ naturally induces an action of $S_{n}$ on $\Omega$. The Johnson graph $J(n, k)$ is an orbital graph which corresponds to the orbital $\left\{(M, N) \in \Omega^{2}|| M \cap N \mid=k-1\right\}$. The distance $i$ graph $J(n, k)_{i}$ of $J(n, k)$ is an orbital graph corresponding to the orbital

$$
\Gamma_{i}=\left\{(M, N) \in \Omega^{2}|| M \cap N \mid=k-i\right\}
$$

(see [6] for orbital graphs).

For a merged Johnson graph $J=J(n, k)_{I}$, the complementation of each $k$-sets $M \rightarrow M^{c}$ induces an isomorphism from $J(n, k)_{I}$ to $J(n, n-k)_{I}$. So we may assume that $k \leq n / 2$. For a merged Johnson graph $J=J(n, k)_{I}$ with $1 \leq k \leq \frac{n}{2}$, if $I=\emptyset$ or $\{1,2, \ldots, k\}$, then $J$ is the null or complete graph and so $\operatorname{Aut}(J)=S_{d}$, where $d=\left(\begin{array}{l}n \\ k\end{array}\right)$. Thus, we further assume that $k \geq 2$ and $\emptyset \varsubsetneqq I \varsubsetneqq\{1,2, \ldots, k\}$. For notational simplicity, let $I^{\prime}=I \backslash\{k\}$, and for any integer $t$, let $t-I=\{t-i \mid i \in I\}$ and $t-I^{\prime}=\left\{t-i \mid i \in I^{\prime}\right\}$. We also denote $I^{\prime \prime}=k-I^{\prime}$, and let $e=\frac{1}{2}\left(\begin{array}{c}n \\ n / 2\end{array}\right)$.

In [9], G. Jones found the automorphism groups of the merged Johnson graphs as follows.

Theorem $3.1([9])$. Let $J=J(n, k)_{I}$, where $2 \leq k \leq \frac{n}{2}$ and $\emptyset \varsubsetneqq I \varsubsetneqq$ $\{1,2, \ldots, k\}$ and let $A=\operatorname{Aut}(J)$.

(1) If $2 \leq k<\frac{n-1}{2}$, and $J \neq J(12,4)_{I}$ with $I=\{1,3\}$ or $\{2,4\}$, then $A=S_{n}$ with orbitals $\Gamma_{0}, \Gamma_{1}, \ldots, \Gamma_{k} \subset \Omega^{2}$.

(2) If $J=J(12,4)_{I}$ with $I=\{1,3\}$ or $\{2,4\}$, then $A=O_{10}^{-1}(2)$ with orbitals $\Gamma_{0}, \Gamma_{1} \cup \Gamma_{3}, \Gamma_{2} \cup \Gamma_{4}$.

(3) If $k=\frac{n-1}{2}$ and $I \neq k+1-I$, then $A=S_{n}$ with orbitals $\Gamma_{0}, \Gamma_{1}, \ldots, \Gamma_{k} \subset$ $\Omega^{2}$.

(4) If $k=\frac{n-1}{2}$ and $I=k+1-I$, then $A=S_{n+1}$ with orbitals $\Gamma_{0}$ and $\Gamma_{i} \cup \Gamma_{k+1-i}$ for all $i=1,2, \ldots,\left\lfloor\frac{k+1}{2}\right\rfloor$.

(5) If $k=\frac{n}{2}$ and $I \neq\{k\}$ nor $\{1,2, \ldots, k-1\}$, and $I^{\prime} \neq I^{\prime \prime}$, then $A=$ $S_{2} \times S_{n}$ with orbitals $\Gamma_{0}, \Gamma_{1}, \ldots, \Gamma_{k} \subset \Omega^{2}$. 
(6) If $k=\frac{n}{2}$ and $I \neq\{k\}$ nor $\{1,2, \ldots, k-1\}$, and $I^{\prime}=I^{\prime \prime}$, then $A=S_{2}^{e}$ : $S_{n}$ with orbitals $\Gamma_{0}$ and $\Gamma_{i} \cup \Gamma_{k-i}$ for all $i=1,2, \ldots,\left\lfloor\frac{k}{2}\right\rfloor$ and $\Gamma_{k}$.

(7) If $k=\frac{n}{2}$ and $I=\{k\}$ or $\{1,2, \ldots, k-1\}$, then $A=S_{2}^{e}: S_{e}=S_{2} 2 S_{e}$ with orbitals $\Gamma_{0}, \Gamma_{1} \cup \cdots \cup \Gamma_{k-1}$ and $\Gamma_{k}$.

To understand the automorphism group $S_{n+1}$ of $J\left(n, \frac{n-1}{2}\right)_{I}$ with $I=k+$ $1-I$, let $[\tilde{n}]=[n] \cup\{\infty\}$ and let $\Psi$ be the set of equipartitions of $[\tilde{n}]$, by which we mean the unordered partitions $\left\{P_{1}, P_{2}\right\}$ of $[\tilde{n}]$ satisfying $\left|P_{1}\right|=\left|P_{2}\right|=\frac{n+1}{2}$. There is a bijection $\phi: \Omega \rightarrow \Psi$, sending each $M$ to $\{M \cup\{\infty\},[n]-M\}$. Note that its inverse sends an equipartition $\left\{P_{1}, P_{2}\right\}$ to $P_{i} \backslash\{\infty\}$, where $i$ is chosen so that $\infty \in P_{i}$. The natural action of $S_{n+1}$ on $[\tilde{n}]$ induces an action of $S_{n+1}$ on $\Omega$. By the condition $I=k+1-I$, one can see that this action induces an automorphism group of $J\left(n, \frac{n-1}{2}\right)_{I}$ (For a detail information, see the paper $[9])$. The next theorem is the main theorem of this paper.

Theorem 3.2. Let $J=J(n, k)_{I}$, where $2 \leq k \leq \frac{n}{2}$ and $\emptyset \varsubsetneqq I \varsubsetneqq\{1,2, \ldots, k\}$.

(1) If $(n, k) \neq(5,2)$ and $J \neq J\left(n, \frac{n}{2}\right)_{I}$ with $I=\left\{\frac{n}{2}\right\},\left\{1,2, \ldots, \frac{n}{2}-1\right\}$ or $I^{\prime}=I^{\prime \prime}$, then $\operatorname{Dist}(J)=2$.

(2) If $J=J(5,2)_{I}$ with $I=\{1\}$ or $\{2\}$; or $J=J\left(n, \frac{n}{2}\right)_{I}$ satisfying $I^{\prime}=I^{\prime \prime}$ and $I$ is neither $\left\{\frac{n}{2}\right\}$ nor $\left\{1,2, \ldots, \frac{n}{2}-1\right\}$, then $\operatorname{Dist}(J)=3$.

(3) If $J=J\left(n, \frac{n}{2}\right)_{I}$ with $I=\left\{\frac{n}{2}\right\}$ or $\left\{1,2, \ldots, \frac{n}{2}-1\right\}$, then $\operatorname{Dist}(J)=$ $\left\lceil\frac{1+\sqrt{1+4\left(\begin{array}{c}n \\ n / 2\end{array}\right)}}{2}\right\rceil$.

Corollary 3.3. Let $G=J(n, k)$ be the Johnson graph with $k \geq 2$.

(1) If $(n, k) \neq(4,2)$ nor $(5,2)$, then $\operatorname{Dist}(G)=2$ and

(2) if $G=J(4,2)$ or $J(5,2)$, then $\operatorname{Dist}(J)=3$.

We will prove Theorem 3.2 in the next section. For the rest of the section, we will prove lemmas which will be used in the proof of Theorem 3.2. For a set $[n]=\{1,2,3, \ldots, n\}$ and for any permutation $\pi$ of $[n], \pi$ can be represented by $\left(i_{1}, i_{2}, \ldots, i_{n}\right)$, where for any $j=1, \ldots, n, \pi(j)=i_{j}$. Throughout the rest of the paper, we use the above representation of permutations. Now for any permutations $\pi=\left(i_{1}, i_{2}, \ldots, i_{n}\right), \sigma=\left(j_{1}, j_{2}, \ldots, j_{n}\right)$ and for any integer $t \in[n]$, $\pi \sigma(t)=\pi(\sigma(t))=\pi\left(j_{t}\right)=i_{j_{t}}$, and hence $\pi \sigma=\left(i_{j_{1}}, i_{j_{2}}, \ldots, i_{j_{n}}\right)$. For any $j \in[n]$, let $\tau_{j}$ be the transposition of $[n]$ exchanging $j$ and $j+1$. Note that for any permutation $\pi=\left(i_{1}, i_{2}, \ldots, i_{n}\right), \pi \tau_{j}=\left(i_{1}, \ldots, i_{j-1}, i_{j+1}, i_{j}, i_{j+2}, \ldots, i_{n}\right)$.

For any permutation $\pi=\left(i_{1}, i_{2}, \ldots, i_{n}\right)$ and for any $k, \ell \in[n]$ with $1 \leq$ $k \leq \frac{n}{2}$, let $V_{k, \ell}^{\pi}$ be the $k$-subset $\left\{i_{\ell}, i_{\ell+1}, \ldots, i_{\ell+k-1}\right\}$, where the subscripts are considered as their residue classes modulo $n$. For our convenience, as a vertex of $J(n, k)_{I}$, we denote $V_{k, \ell}^{\pi}$ by $V_{\ell}^{\pi}$ and if $\pi$ is the identity, we denote $V_{\ell}^{\pi}$ simply by $V_{\ell}$.

For any permutation $\pi=\left(i_{1}, i_{2}, \ldots, i_{n}\right)$, let $\phi_{\pi}$ be the permutation of the vertex set of $J(n, k)_{I}$ defined by

$$
\phi_{\pi}\left(\left\{a_{1}, a_{2}, \ldots, a_{k}\right\}\right)=\left\{\pi\left(a_{1}\right), \pi\left(a_{2}\right), \ldots, \pi\left(a_{k}\right)\right\}=\left\{i_{a_{1}}, i_{a_{2}}, \ldots, i_{a_{k}}\right\}
$$


for any vertex $\left\{a_{1}, a_{2}, \ldots, a_{k}\right\}$ of $J(n, k)_{I}$. Now one can check that $\phi_{\pi}$ be an automorphism of $J(n, k)_{I}$.

Lemma 3.4. For any permutation $\pi=\left(i_{1}, i_{2}, \ldots, i_{n}\right)$ of $[n]$ and for any $\ell \in[n]$, $\phi_{\pi}\left(V_{\ell}\right)=V_{\ell}^{\pi}$. Furthermore for any permutation $\sigma$ of $[n], \phi_{\pi}\left(V_{\ell}^{\sigma}\right)=V_{\ell}^{\pi \sigma}$.

Proof. Since $V_{\ell}=\{\ell, \ell+1, \ldots, \ell+k-1\}$, we have

$$
\phi_{\pi}\left(V_{\ell}\right)=\{\pi(\ell), \pi(\ell+1), \ldots, \pi(\ell+k-1)\}=\left\{i_{\ell}, i_{\ell+1}, \ldots, i_{\ell+k-1}\right\}=V_{\ell}^{\pi} .
$$

Furthermore for any permutation $\sigma$ of $[n]$,

$$
\begin{aligned}
\phi_{\pi}\left(V_{\ell}^{\sigma}\right) & =\phi_{\pi}(\{\sigma(\ell), \sigma(\ell+1), \ldots, \sigma(\ell+k-1)\}) \\
& =\{\pi \sigma(\ell), \pi \sigma(\ell+1), \ldots, \pi \sigma(\ell+k-1)\}=V_{\ell}^{\pi \sigma} .
\end{aligned}
$$

Lemma 3.5. Let $J=J(12,4)_{I}$ with $I=\{1,3\}$. For any permutation $\pi$ of $X$, if an automorphism $\phi$ of $J$ fixes all vertices in $S=\left\{V_{j}^{\pi}, V_{j}^{\pi \tau_{1}}, V_{j}^{\pi \tau_{2}} \mid j=\right.$ $1,2, \ldots, 12\}$, then $\phi$ also fixes $V_{j}^{\pi \tau_{i}}$ for all $i, j=1,2, \ldots, 12$.

Proof. By Lemma 3.4, we may assume that $\pi$ is the identity. Note that $S=$ $\left\{V_{j} \mid j=1,2, \ldots, 12\right\} \cup\left\{V_{2}^{\tau_{1}}, V_{10}^{\tau_{1}}\right\} \cup\left\{V_{3}^{\tau_{2}}, V_{11}^{\tau_{2}}\right\}$, where $V_{2}^{\tau_{1}}=\{1,3,4,5\}, V_{10}^{\tau_{1}}$ $=\{10,11,12,2\}$ and $V_{3}^{\tau_{2}}=\{2,4,5,6\}, V_{11}^{\tau_{2}}=\{11,12,1,3\}$.

Let $\phi$ be an automorphism of $J$ fixing all vertices in $S$. Since $\tau_{3}=(1,2,4,3$, $5,6, \ldots, 12)$, we have $\left\{V_{j}^{\tau_{3}} \mid j=1,2, \ldots, 12\right\} \backslash S=\left\{V_{4}^{\tau_{3}}, V_{12}^{\tau_{3}}\right\}$, where $V_{4}^{\tau_{3}}=$ $\{3,5,6,7\}$ and $V_{12}^{\tau_{3}}=\{12,1,2,4\}$. Let $A=N\left(V_{4}^{\tau_{3}}\right) \cap S$ and $B=N\left(V_{12}^{\tau_{3}}\right) \cap S$. Now we have

$$
A=\left\{V_{1}, V_{3}, V_{4}, V_{5}, V_{7}, V_{12}, V_{11}^{\tau_{2}}\right\} \text { and } B=\left\{V_{1}, V_{3}, V_{4}, V_{9}, V_{11}, V_{12}\right\} .
$$

Let $X$ be a vertex in $J$ such that $N(X) \cap S=A$. For the first case, assume that $\left|X \cap V_{1}\right|=3$. If $X \cap V_{1}=\{1,2,3\}$, then $4,5,6 \notin X$ and $7 \in X$ because $X$ is adjacent to both $V_{3}$ and $V_{4}$ but not to $V_{2}$. In this case, $X$ is adjacent to $V_{6}$, a contradiction. If $X \cap V_{1}=\{1,2,4\}$, then $5,6,7 \notin X$ and $8 \in X$, and hence $X$ is adjacent to $V_{6}$, a contradiction. Similarly, one can show that $X \cap V_{1}$ is neither $\{1,3,4\}$ nor $\{2,3,4\}$. Therefore we have $\left|X \cap V_{1}\right|=1$.

Assume that $X \cap V_{1}=\{1\}$. Then $2,3,4,5 \notin X$ and $6 \in X$ because $V_{2} \notin N(X)$ and $V_{3} \in N(X)$. Since $X$ is adjacent to $V_{4}, V_{5}, V_{7}, V_{12}$ but not to $V_{2}, V_{6}, V_{8}$, the only possible $X$ is $\{1,6,9,11\}$. But $\{1,6,9,11\}$ is adjacent to $V_{2}^{\tau_{1}}=\{1,3,4,5\}$ which is an element in $S \backslash A$, a contradiction. Similarly, one can show that if $X \cap V_{1}=\{2\}$ or $X \cap V_{1}=\{4\}$, then a contradiction occurs. Furthermore when $X \cap V_{1}=\{3\}$, the only possible $X$ is $\{3,5,6,7\}$, which satisfies $N(X) \cap S=A$. Hence $X=\{3,5,6,7\}=V_{4}^{\tau_{3}}$. This implies that $\phi$ also fixes $V_{4}^{\tau_{3}}$.

Let $Y$ be a vertex in $J$ such that $N(Y) \cap S=B$. By considering the fact that $Y$ is adjacent to $V_{1}, V_{3}, V_{4}, V_{9}, V_{11}, V_{12}$ but not to $V_{2}, V_{5}, V_{6}, V_{7}, V_{8}, V_{10}$, one can show that $Y=\{1,6,8,10\},\{2,5,8,9\}$ or $\{12,1,2,4\}$. Since $Y$ is not adjacent to $V_{2}^{\tau_{1}}=\{1,3,4,5\}, Y$ is $V_{12}^{\tau_{3}}=\{12,1,2,4\}$. This implies that $\phi$ fixes $V_{12}^{\tau_{3}}$. Up to now, we showed that $\phi$ fixes $V_{j}^{\tau_{3}}$ for all $j=1,2, \ldots, 12$. 
Since $\phi$ fixes all elements in $\left\{V_{j}, V_{j}^{\tau_{2}}, V_{j}^{\tau_{3}} \mid j=1,2, \ldots, 12\right\}$, one can show that $\phi$ fixes $V_{j}^{\tau_{4}}$ for all $j=1,2, \ldots, 12$ by a similar way. Continuing the similar process, one can show that $\phi$ fixes $V_{j}^{\tau_{i}}$ for all $i, j=1,2, \ldots, 12$.

Lemma 3.6. Let $J=J(12,4)_{I}$ with $I=\{1,3\}$. For any permutation $\pi$ of $[n]$, if an automorphism $\phi$ of $J$ fixes all vertices in $S_{1}=\left\{V_{j}^{\pi}, V_{j}^{\pi \tau_{i}} \mid i, j=\right.$ $1,2, \ldots, 12\}$, then $\phi$ also fixes $V_{j}^{\pi \tau_{i} \tau_{k}}$ for all $i, j, k=1,2, \ldots, 12$.

Proof. By Lemma 3.4, assume that $\pi$ is the identity. Let $\phi$ be an automorphism of $J$ fixing all vertices in $S_{1}$. Note that $\left\{V_{j}^{\tau_{1} \tau_{2}} \mid j=1,2, \ldots, 12\right\}-S_{1}=$ $\left\{V_{3}^{\tau_{1} \tau_{2}}, V_{11}^{\tau_{1} \tau_{2}}\right\}$ because $\tau_{1} \tau_{2}=(2,3,1,4,5, \ldots, 12)$, where $V_{3}^{\tau_{1} \tau_{2}}=\{1,4,5,6\}$ and $V_{11}^{\tau_{1} \tau_{2}}=\{11,12,2,3\}$. Let $C=N\left(V_{3}^{\tau_{1} \tau_{2}}\right) \cap S_{1}$ and $D=N\left(V_{11}^{\tau_{1} \tau_{2}}\right) \cap S_{1}$. Now we have

$$
\begin{aligned}
& \left\{V_{3}, V_{4}, V_{6}, V_{10}, V_{11}, V_{12}, V_{2}^{\tau_{1}}, V_{3}^{\tau_{2}}\right\} \subset C,\left\{V_{1}, V_{2}, V_{5}, V_{7}, V_{8}, V_{9}\right\} \cap C=\emptyset \text { and } \\
& \left\{V_{3}, V_{8}, V_{11}, V_{12}, V_{2}^{\tau_{1}}, V_{3}^{\tau_{2}}\right\} \subset D,\left\{V_{1}, V_{2}, V_{4}, V_{5}, V_{6}, V_{7}, V_{9}, V_{10}\right\} \cap D=\emptyset .
\end{aligned}
$$

Let $X$ be a vertex in $J$ such that $N(X) \cap S_{1}=C$. By considering the fact that $X$ is adjacent to $V_{3}, V_{4}, V_{6}, V_{10}, V_{11}, V_{12}$ but not to $V_{1}, V_{2}, V_{5}, V_{7}, V_{8}, V_{9}$, we have that $X=\{6,8,9,12\},\{1,4,5,6\}$ or $\{2,4,9,10\}$. Since $X$ is adjacent to both $V_{2}^{\tau_{1}}=\{1,3,4,5\}$ and $V_{3}^{\tau_{2}}=\{2,4,5,6\}, X$ is $V_{3}^{\tau_{1} \tau_{2}}=\{1,4,5,6\}$. This implies that $\phi$ also fixes $V_{3}^{\tau_{1} \tau_{2}}$.

Let $Y$ be a vertex in $J$ such that $N(Y) \cap S_{1}=D$. By considering the fact that $Y$ is adjacent to $V_{3}, V_{8}, V_{11}, V_{12}$ but not to $V_{1}, V_{2}, V_{4}, V_{5}, V_{6}, V_{7}, V_{9}, V_{10}$, one can show that $V=\{6,7,10,12\},\{2,4,7,8\}$ or $\{11,12,2,3\}$. Since $Y$ is adjacent to both $V_{2}^{\tau_{1}}=\{1,3,4,5\}$ and $V_{3}^{\tau_{2}}=\{2,4,5,6\}, Y$ is $V_{11}^{\tau_{1} \tau_{2}}=\{11,12,2,3\}$. This implies that $\phi$ fixes $V_{11}^{\tau_{1} \tau_{2}}$. Therefore $\phi$ fixes $V_{j}^{\tau_{1} \tau_{2}}$ for all $j=1,2, \ldots, 12$.

Since $\phi$ fixes all vertices in $\left\{V_{j}^{\pi}, V_{j}^{\pi \tau_{1}}, V_{j}^{\pi \tau_{2}} \mid j=1,2, \ldots, 12\right\}$ with $\pi=\tau_{1}$, $\phi$ also fixes $V_{j}^{\tau_{1} \tau_{i}}$ for all $i, j=1,2, \ldots, 12$ by Lemma 3.5. By a similar way, one can show that $\phi$ fixes $V_{j}^{\tau_{i} \tau_{k}}$ for all $i, j, k=1,2, \ldots, 12$.

Lemma 3.7. For the merged Johnson graph $J=J(12,4)_{I}$ with $I=\{1,3\}$, $S=\left\{V_{j}, V_{j}^{\tau_{1}}, V_{j}^{\tau_{2}} \mid j=1,2, \ldots, 12\right\}$ is a determining set.

Proof. Let $\phi$ be an automorphism of $J$ fixing all vertices in $S=\left\{V_{j}, V_{j}^{\tau_{1}}, V_{j}^{\tau_{2}} \mid\right.$ $j=1,2, \ldots, 12\}$. By Lemma 3.5, $\phi$ fixes $V_{j}^{\tau_{i}}$ for all $i, j=1,2, \ldots, 12$. Furthermore, $\phi$ fixes $V_{j}^{\tau_{i} \tau_{k}}$ for all $i, j, k=1,2, \ldots, 12$ by Lemma 3.6. By applying Lemma 3.6 again with $\pi=\tau_{i}$, one can show that $\phi$ fixes $V_{j}^{\tau_{i} \tau_{k} \tau_{\ell}}$ for all $i, j, k, \ell=1,2, \ldots, 12$. Continuing the similar process, one can show that $\phi$ fixes $V_{j}^{\tau_{i_{1}} \tau_{i_{2}} \cdots \tau_{i_{t}}}$ for any positive integer $t$ and for all $i_{1}, i_{2}, \ldots, i_{t}, j$ in [12]. Since $\left\{\tau_{i} \mid i=1,2, \ldots, 12\right\}$ generates symmetric group on [12], $\phi$ fixes all vertices of $J$, i.e., $S$ is a determining set.

Let $J$ be a merged Johnson graph $J(2 m, m)_{I}$ with $I \subseteq\{1,2, \ldots, m\}$. For any $v \in V(G)$, let $\bar{v}$ be the vertex $[2 m]-v$ for convenience.

Lemma 3.8. Let $J=J(n, k)_{I}$, where $1 \leq k \leq \frac{n}{2}$ and $\emptyset \varsubsetneqq I \varsubsetneqq\{1,2, \ldots, k\}$. 
(1) For $(n, k)=(2 m+1, m)$ with $m \geq 3$ and $I=\{1, m\}, S_{1}=\left\{V_{1}, V_{2}, \ldots\right.$, $\left.V_{m+2}\right\}$ is a determining set.

(2) For $(n, k)=(2 m, m)$ with $m \geq 3$ and $I=\{1\}, S_{2}=\left\{V_{1}, V_{2}, \ldots, V_{2 m}\right\}$ $\cup\{\{1,2, \ldots, m-2, m, m+2\}\}$ is a determining set.

Proof. (1) Note that $\operatorname{Aut}(J)$ is isomorphic to $S_{n+1}$ by Theorem 3.1. For any automorphism $\psi$ of $J$ as a permutation of vertices of $J$, let $\psi^{\prime}$ be a corresponding permutation of $[\tilde{n}]=[n] \cup\{\infty\}$. Let $\phi$ be an automorphism of $J$ fixing all elements in $S_{1}$. Since $\phi$ fixes $V_{1}, \phi^{\prime}$ fixes $\{1,2, \ldots, m, \infty\}$ set-wisely or $\phi^{\prime}$ sends $\{1,2, \ldots, m, \infty\}$ to $\{m+1, m+2, \ldots, 2 m+1\}$ set-wisely.

Case 1: $\phi^{\prime}$ fixes $\{1,2, \ldots, m, \infty\}$ set-wisely.

Since $\phi$ fixes $V_{2}$ and $\phi^{\prime}$ fixes $\{1,2, \ldots, m, \infty\}$ set-wisely, $\phi^{\prime}$ also fixes $\{2,3, \ldots$, $m+1, \infty\}$ set-wisely. This implies that $\phi^{\prime}$ fixes both 1 and $m+1$. Using the fact that $\phi$ fixes all elements in $S_{1}$, one can see that $\phi^{\prime}$ fixes all elements in $[\widetilde{n}]$, namely, $\phi$ is the identity element.

Case 2: $\phi^{\prime}$ sends $\{1,2, \ldots, m, \infty\}$ to $\{m+1, m+2, \ldots, 2 m+1\}$ set-wisely.

Since $\phi$ fixes $V_{2}$ and $\phi^{\prime}$ sends $\{1,2, \ldots, m, \infty\}$ to $\{m+1, m+2, \ldots, 2 m+1\}$ set-wisely, $\phi^{\prime}$ also sends $\{2,3, \ldots, m+1, \infty\}$ to $\{m+2, m+3, \ldots, 2 m+1,1\}$ setwisely. Furthermore this implies that $\phi^{\prime}(\infty)$ belongs to $\{m+2, m+3, \ldots, 2 m+$ $1\}$. By the similar way, one can show that for any $i=1,2, \ldots, m+2, \phi^{\prime}$ sends $\{i, i+1, \ldots, m+i-1, \infty\}$ to $[n]-\{i, i+1, \ldots, m+i-1\}$ set-wisely.

Let $\phi^{\prime}(\infty)=a$. Since $a$ belongs to $\{m+2, \ldots, 2 m+1\}, \phi^{\prime}$ can not send $\{a-m+1, a-m+2, \ldots, a, \infty\}$ to $[n]-\{a-m+1, a-m+2, \ldots, a\}$ set-wisely, which is a contradiction.

Therefore $S_{1}=\left\{V_{1}, V_{2}, \ldots, V_{m+2}\right\}$ is a determining set.

(2) Let $\alpha$ be the automorphism which sends $v$ to $\bar{v}$ for all $v \in V(G)$. Then the order of $\alpha$ is 2 and $\operatorname{Aut}(J) \cong\langle\alpha\rangle \times S_{n}$.

Let $\psi$ be an automorphism of $J$ fixing all elements in $S_{2}$. Since $\psi$ fixes $V_{1}$, $\psi$ is an automorphism induced by a permutation of $[n]$ fixing $\{1,2, \ldots, m\}$ setwisely or $\psi$ is a product of $\alpha$ and an automorphism induced by a permutation of $[n]$ sending $\{1,2, \ldots, m\}$ to $\{m+1, m+2, \ldots, 2 m\}$ set-wisely.

If $\psi$ is an automorphism induced by a permutation of $[n]$, say $\psi_{1}$, fixing $\{1,2, \ldots, m\}$ set-wisely, then $\psi_{1}$ also fixes $\{i, i+1, \ldots, i+m-1\}$ set-wisely for all $i=1,2, \ldots, 2 m$ because $\psi$ fixes $V_{i}$ for all $i=1,2, \ldots, 2 m$. This implies that $\psi$ is the identity. Hence we can assume that $\psi$ is a product of $\alpha$ and an automorphism induced by a permutation of $[n]$, say $\psi_{2}$, sending $\{1,2, \ldots, m\}$ to $\{m+1, m+2, \ldots, 2 m\}$ set-wisely. Since $\psi$ fixes $V_{2}, \psi_{2}$ also sends $\{2,3, \ldots, m+1\}$ to $\{m+2, m+3, \ldots, 2 m, 1\}$ set-wisely. This implies that $\psi_{2}$ exchanges 1 and $m+1$. By a similar way, one can show that $\psi_{2}$ exchanges $i$ and $m+i$ for any $i=1,2, \ldots, m$. But in this case, $\psi$ does not fix $\{1,2, \ldots, m-2, m, m+2\}$. Therefore $S_{2}$ is a determining set.

Lemma 3.9. If $J=J(2 m, m)_{\{1, m-1\}}$ with $m \geq 4$, then $\operatorname{Dist}(J)>2$. 
Proof. For any $v \in V(J)$, let $\beta_{v}$ be the automorphism of $V(J)$ exchanging $v$ and $\bar{v}$ and fixing all other vertices. Let $f: V(J) \rightarrow\{1,2\}$ be a coloring. If there exists $u \in V(J)$ such that $f(u)=f(\bar{u})$, then $\beta_{u}$ is a color-preserving automorphism, and hence $f$ is not 2-distinguishing. Assume that for all $v \in$ $V(J), f(v)$ and $f(\bar{v})$ are distinct. Let $\phi$ be an automorphism of $J$ induced by a non-identity permutation of $[2 m]$. Let

$$
\psi=\left(\prod_{\{u, \bar{u}\} \text { with } f(u) \neq f(\phi(u))} \beta_{u}\right) \phi .
$$

Then $\psi$ is a color-preserving automorphism. Therefore there is no 2-distinguishing coloring, and hence $\operatorname{Dist}(J)>2$.

For $n=2 m$ with $m \geq 4$, let $\Phi$ be the set of equipartitions of $[n]$. Note that the size of $\Phi$ is $\frac{1}{2}\left(\begin{array}{c}n \\ m\end{array}\right)$. The natural action of $S_{n}$ on $[n]$ induces an action of $S_{n}$ on $\Phi$. Let $\pi$ be a permutation in $S_{n}$ satisfying that for all non-identity permutation $\sigma \in S_{n}$, the number of equipartitions in $\Phi$ fixed by $\pi$ is greater than or equal to the number of equipartitions in $\Phi$ fixed by $\sigma$. Suppose that there exist a permutation $\gamma \in S_{n}$ and $i_{1}, i_{2}, \ldots, i_{t} \in[n]$ with $t \geq 3$ such that $\gamma\left(i_{j}\right)=i_{j+1}$ for all $j=1,2, \ldots, t-1$ and $\gamma\left(i_{t}\right)=i_{1}$. Let $\tilde{\gamma} \in S_{n}$ be a permutation that $\tilde{\gamma}\left(i_{2}\right)=i_{1}, \tilde{\gamma}\left(i_{t}\right)=i_{3}$ and $\tilde{\gamma}(\ell)=\gamma(\ell)$ for all $\ell \in[n] \backslash\left\{i_{2}, i_{t}\right\}$. Note that all equipartitions in $\Phi$ fixed by $\gamma$ are also fixed by $\tilde{\gamma}$. Furthermore there is an equipartition fixed by $\tilde{\gamma}$ but not by $\gamma$. This implies that $\pi$ is a product of disjoint transpositions. For two permutations $\gamma_{1}, \gamma_{2} \in S_{n}$, suppose that there exist $i_{1}, i_{2}, \ldots, i_{6} \in[n]$ such that

$$
\begin{aligned}
& \gamma_{1}\left(i_{1}\right)=i_{2}, \gamma_{1}\left(i_{2}\right)=i_{1}, \gamma_{1}\left(i_{3}\right)=i_{4}, \gamma_{1}\left(i_{4}\right)=i_{3}, \gamma_{1}\left(i_{5}\right)=i_{5}, \gamma_{1}\left(i_{6}\right)=i_{6}, \\
& \gamma_{2}\left(i_{1}\right)=i_{2}, \gamma_{2}\left(i_{2}\right)=i_{1}, \gamma_{2}\left(i_{3}\right)=i_{3}, \gamma_{2}\left(i_{4}\right)=i_{4}, \gamma_{2}\left(i_{5}\right)=i_{5}, \gamma_{2}\left(i_{6}\right)=i_{6},
\end{aligned}
$$

and for all $j \in[n] \backslash\left\{i_{1}, i_{2}, \ldots, i_{6}\right\}, \gamma_{1}(j)=\gamma_{2}(j)$. Then one can check that all equipartitions fixed by $\gamma_{1}$ are also fixed by $\gamma_{2}$. This implies that $\pi$ is a transposition or a product of $m$ disjoint transpositions. Note that the number of equipartitions fixed by a transposition is $\left(\begin{array}{c}2 m-2 \\ m-2\end{array}\right)$ and the number of equipartitions fixed by a product of $m$ disjoint transpositions is $2^{m-1}$ if $m$ is odd; $2^{m-1}+\left(\begin{array}{c}m \\ m / 2\end{array}\right)$ if $m$ is even. We aim to show inductively that $\left(\begin{array}{c}2 m-2 \\ m-2\end{array}\right)>2^{m-1}+\left(\begin{array}{c}m \\ m / 2\end{array}\right)$ for all even $m$ with $m \geq 4$. For $m=4$,

$$
\left(\begin{array}{c}
2 m-2 \\
m-2
\end{array}\right)=15>14=2^{m-1}+\left(\begin{array}{c}
m \\
m / 2
\end{array}\right) .
$$

Suppose that for some even $m \geq 4,\left(\begin{array}{c}2 m-2 \\ m-2\end{array}\right)>2^{m-1}+\left(\begin{array}{c}m \\ m / 2\end{array}\right)$. Then

$$
\begin{aligned}
\left(\begin{array}{c}
2 m \\
m-1
\end{array}\right) & =\frac{2 m(2 m-1)}{(m+1)(m-1)}\left(\begin{array}{c}
2 m-2 \\
m-2
\end{array}\right)>2\left(2^{m-1}+\left(\begin{array}{c}
m \\
m / 2
\end{array}\right)\right)>2^{m} \text { and } \\
\left(\begin{array}{c}
2 m+2 \\
m
\end{array}\right) & =\frac{(2 m+2)(2 m+1) 2 m(2 m-1)}{(m+2)(m+1) m(m-1)}\left(\begin{array}{c}
2 m-2 \\
m-2
\end{array}\right)
\end{aligned}
$$




$$
>2^{2} \frac{(2 m+1)(2 m-1)}{(m+2)(m-1)}\left(2^{m-1}+\left(\begin{array}{c}
m \\
m / 2
\end{array}\right)\right)>2^{m+1}+\left(\begin{array}{c}
m+2 \\
m / 2+1
\end{array}\right) .
$$

Hence $\pi$ is a transposition. Furthermore for any non-identity permutation $\sigma \in S_{n}$, the number of equipartitions fixed by $\sigma$ is at most $\left(\begin{array}{c}2 m-2 \\ m-2\end{array}\right)$.

Lemma 3.10. For $n=2 m$ with $m \geq 4$, let $\Phi$ be the set of equipartitions of $[n]$. Now there is a 3-coloring $c: \Phi \rightarrow\{B, R, Y\}$ of $\Phi$ such that only identity permutation in $S_{n}$ preserves all colors under the induced action of $S_{n}$ on $\Phi$.

Proof. Give a random coloring on $\Phi$ with three colors $\{B, R, Y\}$. For any nonidentity permutation $\sigma$ of $[2 \mathrm{~m}]$, let $A_{\sigma}$ be the event that $\sigma$ preserves colors of all equipartitions of $[2 m]$. Note that the number of equipartitions fixed by $\sigma$ is at most $\left(\begin{array}{c}2 m-2 \\ m-2\end{array}\right)$. Namely, the number of equipartitions which are not fixed by $\sigma$ is at least

$$
|\Phi|-\left(\begin{array}{c}
2 m-2 \\
m-2
\end{array}\right)=\frac{1}{2}\left(\begin{array}{c}
2 m \\
m
\end{array}\right)-\left(\begin{array}{c}
2 m-2 \\
m-2
\end{array}\right)=\frac{m}{m-1}\left(\begin{array}{c}
2 m-2 \\
m-2
\end{array}\right) .
$$

For any orbit $O$ of $\sigma$ whose size is $t$ with $t \geq 2$ under the action of $S_{2 m}$ on $\Phi$, the probability that $\sigma$ preserves colors of all equipartitions in $O$ is $3^{-t+1}$, which is less than $3^{-\frac{t}{2}}$. Hence we have

$$
\operatorname{Pr}\left(A_{\sigma}\right) \leq 3^{-\frac{m}{2(m-1)}}\left(\begin{array}{c}
2 m-2 \\
m-2
\end{array}\right) .
$$

Therefore

$$
\begin{aligned}
\operatorname{Pr}\left(\bigcup_{\sigma \in S_{n} \backslash\{i d\}} A_{\sigma}\right) \leq \sum_{\sigma \in S_{n} \backslash\{i d\}} \operatorname{Pr}\left(A_{\sigma}\right) & <((2 m) !-1) 3^{-\frac{m}{2(m-1)}}\left(\begin{array}{c}
2 m-2 \\
m-2
\end{array}\right) \\
& \leq(2 m) ! 3^{-\frac{m}{2(m-1)}\left(\begin{array}{c}
2 m-2 \\
m-2
\end{array}\right)}
\end{aligned}
$$

where $i d$ is the identity permutation of $[n]$. For $n=8$, the number

$$
n ! 3^{-\frac{m}{2(m-1)}}\left(\begin{array}{c}
2 m-2 \\
m-2
\end{array}\right)
$$

is $\frac{4480}{3^{8}}$, and it is less than 1 . Furthermore for any $m \geq 4$,

$$
\frac{(2 m+2) ! 3^{-\frac{m+1}{2 m}\left(\begin{array}{c}
2 m \\
m-1
\end{array}\right)}}{(2 m) ! 3^{-\frac{m}{2(m-1)}\left(\begin{array}{c}
2 m-2 \\
m-2
\end{array}\right)}}=\frac{(2 m+2)(2 m+1)}{3^{\frac{3 m-2}{2(m-1)}\left(\begin{array}{c}
2 m-2 \\
m-2
\end{array}\right)}}<\frac{(2 m+2)(2 m+1)}{3^{\frac{3}{2}\left(\begin{array}{c}
2 m-2 \\
m-2
\end{array}\right)}}<1
$$

because

$$
\begin{aligned}
3^{\frac{3}{2}\left(\begin{array}{c}
2 m-2 \\
m-2
\end{array}\right)} & =3^{\frac{3(2 m-2)(2 m-3) \cdots(m+1)}{2 \cdot(m-2) !}}=3^{\frac{12(m-1)(m-2)(2 m-3)(2 m-5)(2 m-6) \cdots(m+1)}{12(m-2)(m-3)(m-4) \cdots 5 \cdot 4}} \\
& =3^{\frac{(m-1)(2 m-3)(2 m-5)(2 m-6) \cdots(m+1)}{(m-3)(m-4) \cdots 5 \cdot 4}} \\
& >3^{(m-1)(2 m-3)(2 m-5)}>(2 m+2)(2 m+1) .
\end{aligned}
$$

This implies that for any $m \geq 4$,

$$
\operatorname{Pr}\left(\bigcup_{\sigma \in S_{n} \backslash\{i d\}} A_{\sigma}\right)<1
$$




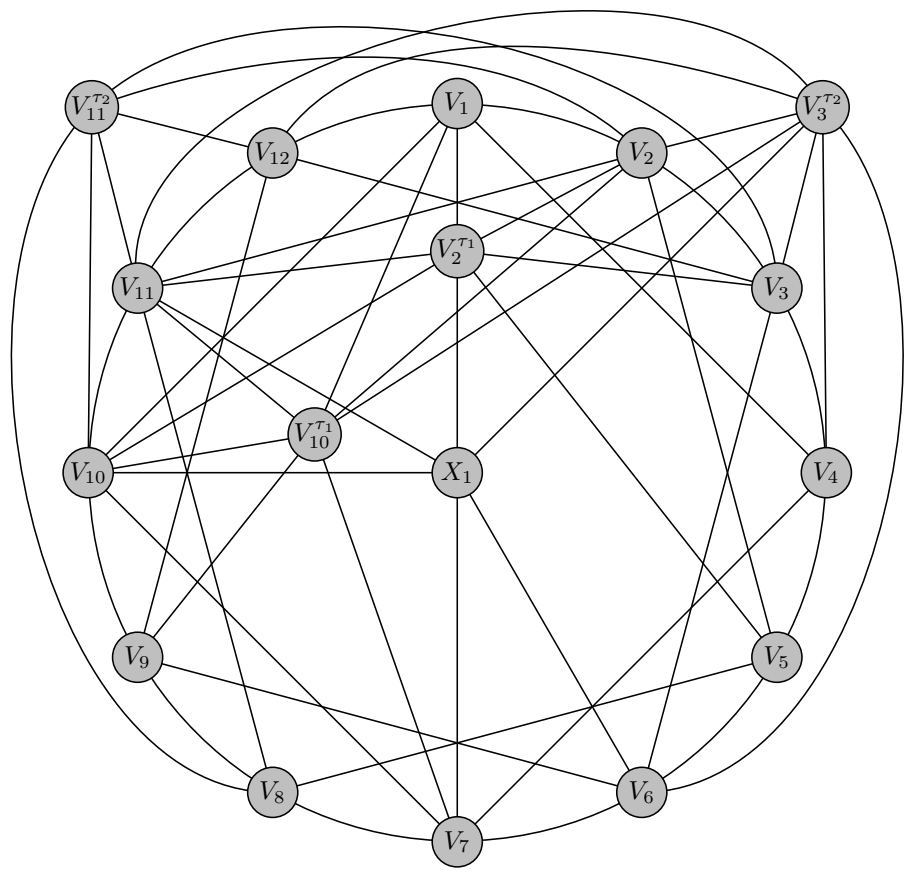

FIGURE 1. An asymmetric subgraph induced by a determining set of $J(12,4)_{\{1,3\}}$.

and hence there exists a 3-coloring $c: \Phi \rightarrow\{B, R, Y\}$ such that the identity permutation in $S_{2 m}$ is the only color-preserving permutation under the induced action of $S_{2 m}$ on $\Phi$.

\section{A proof of the main theorem}

In this section, we prove Theorem 3.2 which is the main result in this paper. For any positive integer $i$, let $D_{i}$ be the set of all vertices whose degrees are $i$.

Let $J=J(n, k)_{I}$, where $2 \leq k \leq \frac{n}{2}$ and $\emptyset \varsubsetneqq I \varsubsetneqq\{1,2, \ldots, k\}$.

Case 1: $(n, k) \neq(5,2)$ and $2 \leq k<\frac{n-1}{2}$, and $J \neq J(12,4)_{I}$ with $I=\{1,3\}$ or $\{2,4\}$.

In this case, $\operatorname{Aut}(J)=\operatorname{Aut}(K(n, k))$ as an acting group on the vertex set, and hence $\operatorname{Dist}(J)=\operatorname{Dist}(K(n, k))=2$ by Proposition 2.3 and Lemma 2.4.

Case 2: $J=J(12,4)_{I}$ with $I=\{1,3\}$ or $\{2,4\}$.

Assume that $J=J(12,4)_{I}$ with $I=\{1,3\}$. Let $S_{1}=\left\{V_{j}, V_{j}^{\tau_{1}}, V_{j}^{\tau_{2}} \mid j=\right.$ $1,2, \ldots, 12\} \cup\left\{X_{1}=\{1,3,5,7\}\right\}$ and let $H_{1}$ be the subgraph of $J$ induced by $S_{1}$ as illustrated in Figure 1. Then $S_{1}$ is a determining set by Lemma 3.7. Let 


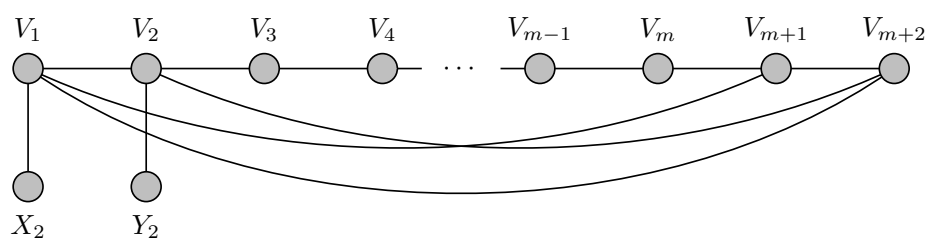

FIGURE 2. An asymmetric subgraph induced by a determining set of $J(2 m+1, m)_{\{1, m\}}$.

$\psi_{1}$ be an automorphism of $H_{1}$. Note that the order of $H_{1}$ is 17 and

$$
\begin{aligned}
& D_{5}=\left\{V_{4}, V_{5}, V_{8}, V_{9}\right\}, D_{6}=\left\{V_{1}, V_{6}, V_{7}, V_{12}, V_{11}^{\tau_{2}}, X_{1}\right\}, \\
& D_{7}=\left\{V_{3}, V_{2}^{\tau_{1}}, V_{10}^{\tau_{1}}\right\}, D_{8}=\left\{V_{2}, V_{10}, V_{3}^{\tau_{2}}\right\}, D_{9}=\left\{V_{11}\right\} .
\end{aligned}
$$

Since $D_{9}=\left\{V_{11}\right\}, \psi_{1}\left(V_{11}\right)=V_{11}$. The fact $N\left(V_{11}\right) \cap D_{5}=\left\{V_{8}\right\}$ implies that $\psi_{1}\left(V_{8}\right)=V_{8}$. Since $N\left(V_{8}\right) \cap D_{5}=\left\{V_{5}, V_{9}\right\}, V_{4}$ is the only vertex in $D_{5}$ which is not contained to $N\left(V_{8}\right) \cup\left\{V_{8}\right\}$. Hence $\psi_{1}$ fixes $V_{4}$. By the fact that $N\left(V_{4}\right) \cap N\left(V_{8}\right) \cap D_{5}=\left\{V_{5}\right\}, \psi_{1}$ fixes both $V_{5}$ and $V_{9}$. Note that $N\left(V_{8}\right) \cap$ $N\left(V_{11}\right)=\left\{V_{11}^{\tau_{2}}\right\}$. So $\psi_{1}$ fixes $V_{11}^{\tau_{2}}$. The fact $N\left(V_{4}\right) \cap N\left(V_{8}\right) \cap D_{6}=\left\{V_{7}\right\}$ and $N\left(V_{5}\right) \cap N\left(V_{9}\right) \cap D_{6}=\left\{V_{6}\right\}$ implies that $\psi_{1}$ fixes both $V_{6}$ and $V_{7}$. Furthermore $\psi_{1}$ fixes $X_{1}=\{1,3,5,7\}$ because $N\left(V_{6}\right) \cap N\left(V_{7}\right)=\left\{X_{1}\right\}$. Since $N\left(V_{7}\right) \cap$ $N\left(V_{11}\right) \cap D_{7}=\left\{V_{10}^{\tau_{1}}\right\}$ and $N\left(V_{7}\right) \cap N\left(V_{11}\right) \cap D_{8}=\left\{V_{10}\right\}, \psi_{1}$ also fixes both $V_{10}$ and $V_{10}^{\tau_{1}}$. Note that $N\left(V_{4}\right) \cap N\left(V_{6}\right) \cap D_{7}=\left\{V_{3}\right\}$ and $N\left(V_{4}\right) \cap N\left(V_{6}\right) \cap D_{8}$ $=\left\{V_{3}^{\tau_{2}}\right\}$. This implies that $\psi_{1}\left(V_{3}\right)=V_{3}$ and $\psi_{1}\left(V_{3}^{\tau_{2}}\right)=V_{3}^{\tau_{2}}$. By the fact $N\left(V_{5}\right) \cap N\left(V_{11}\right)=\left\{V_{2}^{\tau_{1}}\right\}$ and $N\left(V_{9}\right) \cap N\left(V_{11}^{\tau_{2}}\right) \cap D_{6}=\left\{V^{12}\right\}, \psi_{1}$ fixes both $V_{2}^{\tau_{1}}$ and $V_{12}$. Up to now, we know that $\psi_{1}$ fixes all vertices in $V(H)$ except $V_{1}$ and $V_{2}$. Since the degree of $V_{1}$ is 6 and that of $V_{2}$ is $8, \psi_{1}$ also fixes both $V_{1}$ and $V_{2}$. Therefore $\psi_{1}$ is the identity, which implies that $H$ is an asymmetric graph. By Corollary 2.2, we have $\operatorname{Dist}(J)=2$.

For any $J_{1}=J(12,4)_{I}$ with $I=\{2,4\} \operatorname{Aut}\left(J_{1}\right)=\operatorname{Aut}(J)$. Therefore $\operatorname{Dist}\left(J_{1}\right)=\operatorname{Dist}(J)=2$.

Case 3: $J=J(5,2)_{I}$ with $I=\{1\}$ or $\{2\}$.

Since $J(5,2)_{\{2\}}$ is the Kneser graph $K(5,2)$ and $J(5,2)_{\{1\}}$ is its complement, we have $\operatorname{Dist}(J)=3$ by Proposition 2.3 .

Case 4: $k=\frac{n-1}{2}$ and $I \neq k+1-I$.

If $n=5$, then $I=\{1\}$ or $\{2\}$, which means that $I=k+1-I$. Hence we may assume that $n \geq 7$. In this case, since $\operatorname{Aut}(J)=\operatorname{Aut}\left(K\left(n, \frac{n-1}{2}\right)\right)$ as an acting group on the vertex set, one can find that $\operatorname{Dist}(J)=\operatorname{Dist}(K(n, k))=2$ by Proposition 2.3 and Lemma 2.4 .

Case 5: $k=\frac{n-1}{2}$ and $I=k+1-I$.

Let $J=J(2 m+1, m)_{\{1, m\}}$ with $m \geq 3$. Let

$$
S_{2}=\left\{V_{1}, V_{2}, \ldots, V_{m+2}\right\} \cup\left\{X_{2}, Y_{2}\right\},
$$




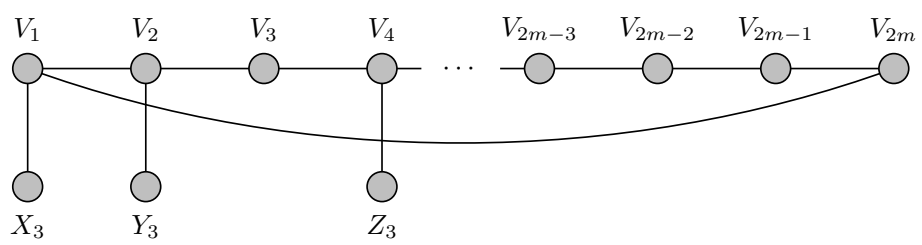

FiguRE 3 . An asymmetric subgraph induced by a determining set of $J(2 m, m)_{\{1\}}$.

where $X_{2}=\{1,2, \ldots, m-2, m, m+2\}$ and $Y_{2}=\{2,3, \ldots, m-1, m+1, m+3\}$; and let $H_{2}$ be the subgraph of $J$ induced by $S_{2}$ as depicted in Figure 2. Now $S_{2}$ is a determining set of $J$ by Lemma 3.8(1). Let $\psi_{2}$ be an automorphism of $\mathrm{H}_{2}$. Note that

$D_{1}=\left\{X_{2}, Y_{2}\right\}, D_{2}=\left\{V_{3}, V_{4}, \ldots, V_{m}\right\}, D_{3}=\left\{V_{m+1}, V_{m+2}\right\}, D_{4}=\left\{V_{1}, V_{2}\right\}$.

Since $V_{1}$ is the only vertex adjacent to all vertices in $D_{3}, \psi_{2}\left(V_{1}\right)=V_{1}$. This implies that $\psi_{2}$ fixes $V_{2}, X_{2}$ and $Y_{2}$ because $D_{4}=\left\{V_{1}, V_{2}\right\}$; and $X_{2}$ and $Y_{2}$ are only adjacent to $V_{1}$ and $V_{2}$, respectively. By the fact that $V_{m+2}$ is the only vertex adjacent to all vertices in $D_{4}, \psi_{2}\left(V_{m+2}\right)=V_{m+2}$. This implies that $\psi_{2}$ also fixes $V_{m+1}$ because $D_{3}=\left\{V_{m+1}, V_{m+2}\right\}$. Since $N\left(V_{2}\right) \cap D_{2}=\left\{V_{3}\right\}$, $\psi_{2}\left(V_{3}\right)=V_{3}$. By a similar way, one can show that $\psi_{2}$ fixes all vertices in $H_{2}$, and hence $\psi_{2}$ is the identity. This means that $H_{2}$ is asymmetric. By Corollary 2.2 , we have $\operatorname{Dist}(J)=2$.

For any $J_{2}=J(2 m+1, m)_{I}$ satisfying $I=k+1-I$, $\operatorname{Aut}\left(J_{2}\right)=\operatorname{Aut}(J)$. Therefore $\operatorname{Dist}\left(J_{2}\right)=\operatorname{Dist}(J)=2$.

Case 6: $k=\frac{n}{2}$ and $I$ is neither $\{k\}$ nor $\{1,2, \ldots, k-1\}$, and $I^{\prime} \neq I^{\prime \prime}$.

Let $J=J(2 m, m)_{\{1\}}$ with $m \geq 3$. Let

$$
S_{3}=\left\{V_{1}, V_{2}, \ldots, V_{2 m}\right\} \cup\left\{X_{3}, Y_{3}, Z_{3}\right\},
$$

where $X_{3}=\{1,2, \ldots, m-2, m, m+2\}, Y_{3}=\{2,3, \ldots, m-1, m+1, m+3\}$ and $Z_{3}=\{4,5, \ldots, m+1, m+3, m+5\}$; and let $H_{3}$ be the subgraph of $J$ induced by $S_{3}$ as shown in Figure 3. Now $S_{3}$ is a determining set of $J$ by Lemma 3.8(2). Let $\psi_{3}$ be an automorphism of $H_{3}$. Note that

$$
D_{1}=\left\{X_{3}, Y_{3}, Z_{3}\right\}, D_{3}=\left\{V_{1}, V_{2}, V_{4}\right\} \text { and } D_{2}=V\left(H_{3}\right)-\left(D_{1} \cup D_{3}\right) \text {. }
$$

By a similar way with cases 2 and 5 , one can show that $\psi_{3}$ fixes all vertices in $H_{3}$, and hence $\psi_{3}$ is the identity. So $H_{3}$ is an asymmetric graph. By Corollary 2.2 , we have $\operatorname{Dist}(J)=2$.

For any $J_{3}=J(2 m, m)_{I}$ satisfying $I \neq\{k\}$ nor $\{1,2, \ldots, k-1\}$, and $I^{\prime} \neq I^{\prime \prime}$, $\operatorname{Aut}\left(J_{3}\right)=\operatorname{Aut}(J)$. Therefore we have $\operatorname{Dist}\left(J_{3}\right)=\operatorname{Dist}(J)=2$.

Case 7: $k=\frac{n}{2}, I^{\prime}=I^{\prime \prime}$ and $I$ is neither $\{k\}$ nor $\{1,2, \ldots, k-1\}$.

Note that for $n \leq 6$, this case can not occur. Hence assume that $n \geq 8$. Let $J=J(2 m, m)_{\{1, m-1\}}$ with $m \geq 4$. By Lemma 3.9, we have $\operatorname{Dist}(J) \geq 3$. Let 
$f: V(J) \rightarrow\{1,2,3\}$ be a random coloring satisfying that for any $u \in V(J)$, $f(u)$ and $f(\bar{u})$ are distinct. Note that for any $u \in V(J)$,

$$
\begin{aligned}
\operatorname{Pr}(\{f(u), f(\bar{u})\}=\{1,2\}) & =\operatorname{Pr}(\{f(u), f(\bar{u})\}=\{1,3\}) \\
& =\operatorname{Pr}(\{f(u), f(\bar{u})\}=\{2,3\})=\frac{1}{3} .
\end{aligned}
$$

Let $\Phi$ be the set of equipartitions of $[n]$. Using a random coloring $f$ : $V(J) \rightarrow\{1,2,3\}$, we can define the coloring $\tilde{f}: \Phi \rightarrow\{B, R, Y\}$ as follows: for any $\{u, \bar{u}\} \in \Phi$, let $\tilde{f}(\{u, \bar{u}\})=B$ if $\{f(u), f(\bar{u})\}=\{1,2\}$; let $\tilde{f}(\{u, \bar{u}\})=R$ if $\{f(u), f(\bar{u})\}=\{1,3\}$; and let $\tilde{f}(\{u, \bar{u}\})=Y$ if $\{f(u), f(\bar{u})\}=\{2,3\}$. Then we can consider $\tilde{f}$ as a random 3 -coloring of $\Phi$. By Lemma 3.10, there is a 3-coloring $f: V(J) \rightarrow\{1,2,3\}$ such that only the identity permutation in $S_{n}$ preserves all colors of equipartitions in its corresponding 3-coloring $\tilde{f}$ : $\Phi \rightarrow\{B, R, Y\}$. This implies that there exists a 3 -distinguishing coloring $f: V(J) \rightarrow\{1,2,3\}$. Therefore $\operatorname{Dist}(J) \leq 3$, and hence $\operatorname{Dist}(J)=3$.

For any $J_{4}=J(2 m, m)_{I}$ satisfying $I^{\prime}=I^{\prime \prime}$ and $I$ is neither $\{k\}$ nor $\{1,2, \ldots$, $k-1\}, \operatorname{Aut}\left(J_{4}\right)=\operatorname{Aut}(J)$. Therefore $\operatorname{Dist}\left(J_{4}\right)=\operatorname{Dist}(J)=3$.

Case $8: k=\frac{n}{2}$ and $I=\{k\}$ or $\{1,2, \ldots, k-1\}$.

Let $J=J(2 m, m)_{\{m\}}$. Then $J$ is composed of $\frac{\left(\begin{array}{c}2 m \\ m\end{array}\right)}{2}$ components which are isomorphic to $K_{2}$. Note that a coloring $f: V(J) \rightarrow\{1,2, \ldots, r\}$ is an $r$-distinguishing if and only if for any vertex $u \in V(J), f(u)$ and $f(\bar{u})$ are distinct and for any two vertex $v, w \in V(J)$ contained to different components, $\{f(v), f(\bar{v})\} \neq\{f(w), f(\bar{w})\}$. Hence Dist $(J)$ is the smallest integer $r$ such that $\left(\begin{array}{l}r \\ 2\end{array}\right) \geq \frac{\left(\begin{array}{c}2 m \\ m\end{array}\right)}{2}$. Therefore $\operatorname{Dist}(J)=\left\lceil\frac{1+\sqrt{1+4\left(\begin{array}{c}2 m \\ m\end{array}\right)}}{2}\right\rceil$.

For any $J_{5}=J(2 m, m)_{I}$ with $I=\{1,2, \ldots, m-1\}, J_{5}$ is the complement of $J$. Hence $\operatorname{Dist}\left(J_{5}\right)=\operatorname{Dist}(J)=\left\lceil\frac{1+\sqrt{1+4\left(\begin{array}{c}2 m \\ m\end{array}\right)}}{2}\right\rceil$.

Acknowledgments. We would like to thank the referees of this paper for valuable comments and suggestions.

\section{References}

[1] M. O. Albertson and D. L. Boutin, Distinguishing geometric graphs, J. Graph Theory 53 (2006), no. 2, 135-150.

[2] — Using determining sets to distinguish Kneser graphs, Electron. J. Combin. 14 (2007), no. 1, Research paper 20, 9 pp.

[3] M. O. Albertson and K. L. Collins, Symmetry breaking in graphs, Electron. J. Combin. 3 (1996), no. 1, Research paper 18, $17 \mathrm{pp}$

[4] B. Bogstad and L. J. Cowen, The distinguishing number of the hypercube, Discrete Math. 283 (2004), no. 1-3, 29-35.

[5] M. Chan, The distinguishing number of the direct product and wreath product action, J. Algebraic Combin. 24 (2006), no. 3, 331-345.

[6] J. Dixon and B. Mortimer, Permutation Groups, Springer, GTM 163, 1996.

[7] M. J. Fisher and G. Isaak, Distinguishing colorings of Cartesian products of complete graphs, Discrete Math. 308 (2008), no. 11, 2240-2246. 
[8] W. Imrich and S. Klavzar, Distinguishing Cartesian powers of graphs, J. Graph Theory 53 (2006), no. 3, 250-260

[9] G. A. Jones, Automorphisms and regular embeddings of merged Johnson graphs, European J. Combin. 26 (2005), no. 3-4, 417-435.

[10] S. Klavzar and X. Zhu, Cartesian powers of graphs can be distinguished by two labels, European J. Combin. 28 (2007), no. 1, 303-310.

[11] T. L. Wong and X. Zhu, Distinguishing labeling of group actions, Discrete Math. 309 (2009), no. 6, 1760-1765.

[12] T. Van Zandt, PSTricks: PostScript macros for generic $T_{E} X$, Available at ftp:// ftp.princeton.edu/pub/tvz/.

DONGSEOK KIM

Department of Mathematics

KYONGGI UNIVERSITY

Suwon 443-760, Korea

E-mail address: dongseok@kgu.ac.kr

Young Soo Kwon

Department of Mathematics

Yeungnam University

KyONGSAN 712-749, Korea

E-mail address: ysookwon@ynu.ac.kr

JAEUn LeF

Department of Mathematics

YeungNAM UNIVERSITY

KYONGSAN 712-749, KoREA

E-mail address: julee@yu.ac.kr 